\title{
MULTISCALE FRAMEWORK FOR MODELING AND ANALYZING LIGHT INTERCEPTION BY TREES
}

\author{
DA SILVA DAVID ${ }^{\dagger}$, BOUDON FRÉDÉRIC $^{2, \dagger}$, GODIN CHRISTOPHE $^{2, \dagger}$, SINOQUET $^{2}$ \\ HERVÉ ${ }^{3, \ddagger}$ \\ ${ }^{1}$ CIRAD, Avenue Agropolis, 34398 Montpellier Cedex 5, France \\ 2 INRIA, 2004 route des lucioles BP 93, 06902 Sophia Antipolis, France \\ 3 INRA, Site de Crouël, 234 avenue du Brézet, 63100 Clermont-Ferrand, France \\ $\dagger$ Virtual Plants, UMR DAP, TA A-96/02, 34398 Montpellier Cedex 5, France \\ $\ddagger$ UMR PIAF, Site de Crouël, 234 avenue du Brézet, 63100 Clermont-Ferrand, France
}

\begin{abstract}
This paper presents a new framework for modeling light interception by isolated trees which makes it possible to analyze the influence of structural tree organization on light capture. The framework is based on a multiscale representation of the plant organization. Tree architecture is decomposed into a collection of components representing clusters of leaves at different scales in the tree crown. The components are represented by porous envelopes automatically generated as convex hulls containing components at a finer scale. The component opacity is defined as the interception probability of a light beam going through its envelope. The role of tree organization on light capture was assessed by running different scenarii where the components at any scale were either randomly distributed or localized to their actual 3D position. The modeling framework was used with threedimensional digitized fruit trees, namely peach and mango trees. A sensitivity analysis was carried out to assess the effect of the spatial organization in each scale on light interception. This modeling framework makes it possible to identify a level of tree description that achieves a good compromise between the amount of measurement required to describe the tree architecture and the quality of the resulting light interception model.
\end{abstract}

Key words. multiscale modeling, light interception, three-dimensional (3-D) modeling, functionalstructural modeling, porous envelope, clumping, fruit trees

1. Introduction. Light capture by plants is an essential process for plant growth and survival. Indeed light provides plants with energy which can be used for carbon fixation through foliage photosynthesis and for transpiration which allows water and nutriment transport within the plant [19]. Light interception by plants foliage is governed by simple basic principles: photons coming from the sun direction (direct radiation) and the whole sky hemisphere (diffuse radiation) may be intercepted by the plant elements or transmitted onto the soil surface if they pass in the foliage gaps. Intercepted photons may then be absorbed or scattered in any direction. Scattered photons may then either be intercepted again or leave the canopy.

The interception process can be seen as the intersection between the photon trajectory - a line - and the plant organ. It thus only depends on the canopy structure, i.e. the spatial distribution of the plant organs, and organ geometry, namely shape, size and orientation [36]. If the detailed three-dimensional (3D) geometry is known, light interception can be easily and accurately computed by using $3 \mathrm{D}$ computer plant mock-up. Ray-tracing methods [13], Z-buffer approach [13] or plant image processing [41] can be used. Although accurate, this class of computation methods shows several shortcomings. First, it does not allow one understanding which structural features are the main determinants of light interception by plants. Second, although methods exist to exhaustively measure the detailed 3D geometry - in particular 3D digitizing [38] -, these techniques are very tedious and do not allow describing large sets of large trees. Third if scattering is computed, the algorithm complexity dramatically increases due to multiple interception-scattering events and the high number of traced 
photons needed for convergence. Fourth, even though the basic processes are computed without any assumption, the simulation results are sensitive to measurement errors in the detailed canopy structure and in the optical properties of plant organs.

For more than 50 years, more simple approaches have been proposed to estimate light interception by plants [23]. The most common approach abstracts the plant canopy as a turbid medium [36], i.e. a medium made of infinitely small foliage particles randomly dispersed in the vegetation volume and thus having a uniform optical density, i.e. transparency. In such medium, light penetration can be expressed by the Beer-Lambert law, i.e. the probability that a photon crosses the vegetation volume without any interception can be written as

$$
p_{0}=\exp (-G \cdot L A D \cdot \mathcal{L}) .
$$

Hence, $p_{0}$ is the probability of zero-interception and corresponds to the canopy porosity. $L A D$ is the leaf area density and $G$ is the extinction coefficient, namely the projection coefficient of plant elements on a plane perpendicular to the direction $\Omega$ [36]. $G$ depends on the angle between $\Omega$ and the normal of the plants elements. The product G.LAD can be regarded as the optical density of the vegetation. Note that leaves are usually the only elements taken into account because they represent the solar collector of the plant. Finally $\mathcal{L}$ is the distance travelled by the photons in the canopy. If scattering is disregarded which is the case in this study where we focus on the effect of canopy structure on light interception, the intercepted light is proportional to $p=1-p_{0}$, i.e. the probability of light interception that defines the canopy opacity.

For a photon direction $\Omega$, the distance $\mathcal{L}$ is constant for horizontally homogeneous vegetation canopies, e.g. grasslands. However for tree crowns, $\mathcal{L}$ depends on the point where the photons enter the canopy. Usually, tree crowns are abstracted by envelopes and beams are sent from a grid of points above the tree. The contribution of each beam to light interception is then summed up to compute total light interception probability in this direction. Finally the contribution of each direction $\Omega$ is summed up by weighting each directional probability with the fraction of incident radiation coming from direction $\Omega$. Several light interception models for tree crowns are based on these principles $[8,22]$. However the assumption of uniform random distribution of leaf elements is rarely verified in actual tree crowns $[46,11,38]$. Indeed leaves are grouped around shoots, with higher density at the crown periphery. This leads to an overall clumped dispersion of the foliage, non-uniform $L A D$ distribution and lesser interception by crowns showing foliage clumping. The simplest way to deal with the non-random location of leaf elements is to introduce a leaf dispersion parameter $\mu$ in the Beer-Lambert equation

$$
p_{0}=\exp (-G \cdot \mu \cdot L A D \cdot \mathcal{L}) .
$$

Parameter $\mu$ equals 1 for random distribution. It is lesser than 1 for clumped foliage, i.e. crown porosity, $p_{0}$, is higher, and $\mu$ could be greater than 1 if foliage would show regular dispersion. Indeed the product $\mu . L A D$ can be regarded as the $L A D$ of equivalent random canopy showing the same porosity. Thus a clumped canopy shows a higher porosity and therefore a lower equivalent $L A D$, i.e. $\mu<1$.

The parameter $\mu$ possibly changes with direction $\Omega[27]$ and botanical parameters $[26,6]$. However there is not yet a clear knowledge about the structural parameters 
determining the degree of foliage clumping, although Sinoquet et al. [40] have showed that $\mu$ is related to the spatial variations of $L A D$.

Two other approaches have been proposed to deal with non-random and nonuniform foliage. In the first one, the crown volume is divided into voxels, and a value of $L A D$ is assigned to each voxel [20, 10,25]. This approach shows two shortcomings. On the one hand, computed light interception depends on voxel size [21,39]. On the other hand, assigning $L A D$ values to each voxel needs a huge amount of field measurements [10]. The second approach is based on the botanical multiscale structure of trees, and was applied to conifer species. Norman and Jarvis [28] assumed that spruce crowns were made of whorls regularly distributed along the trunk, with shoots randomly distributed in whorls and needles randomly dispersed in shoots. Oker-Blom and Kellomaki [30] proposed a simplification of Norman and Jarvis' approach, where shoots were directly distributed at random in the tree crown of Scots pines. This kind of model, called grouping model, better takes into account the foliage distribution according to the plant organization at several scales. It allows better rendering of foliage clumping without introducing a calibration parameter $\mu$.

The objective of this study was to develop a general modeling framework for computing light interception by single tree crowns. This framework includes most of the previously proposed methods in a unifying formalism: 3D plant mock-ups vs. turbid medium, mono- vs. multiscale approaches. This modeling framework is aimed at better understanding the effects of the crown organization on light capture at the whole tree scale, i.e. giving meaning to $\mu$. The expected outcome of this study is to define ways of describing canopy structure as simple as possible and allowing accurate estimation of light interception, without the need of introducing any empirical dispersion parameter $\mu$. In this paper, this approach was applied to a collection of fruit trees, namely four peach and four mango trees.

2. Modelling framework. At a macroscopic level, the problem consists of estimating the amount of direct radiation intercepted by a vegetal component $x$ (representing either the entire plant crown or a sub-branching system) for each direction $\Omega$ of incident light. Light interception is computed in terms of $S T A R$, Silhouette to Total Area Ratio:

$$
S T A R \triangleq \frac{P L A}{T L A},
$$

where $P L A\left(\mathrm{~m}^{2}\right)$ is the Projected Leaf Area on a plane perpendicular to the incident direction $\Omega$ (i.e. the leaf area which intercepts light in direction $\Omega$ ) and $T L A\left(m^{2}\right)$ is the total leaf area [5, 31]. The $S T A R$ is thus the relative irradiance of leaf area. To take into account the clumping of leaves in plant crowns, this definition can be extended to the case where a canopy is decomposed into groups of leaves rather than directly into leaves. Groups of leaves can be regarded as macroscopic plant components, corresponding for instance to particular branching systems in the plant. In this case, the notion of $P L A$ must be redefined since it now refers to the projected area of these coarser components, which are not entirely opaque. For this, we assume that the shape of a component $x$ can be globally characterized by its convex envelope. According to [18], the $P L A$ of $x$, denoted $P L A_{x}$, can then be defined from the projected surface of the component envelope by introducing its opacity $p_{x}$ in direction $\Omega$ [40] 


$$
P L A_{x} \triangleq P E A_{x} \cdot p_{x},
$$

where $P E A_{x}$ is the Projected Envelope Area of $x$ in the direction $\Omega$ and $p_{x}$ can be regarded as the probability of photon interception in the projected envelope. Reciprocally, $1-p_{x}$ is the envelope porosity. In the case of such multiscale plant structures, our original question thus amounts to estimating the opacity of the coarse components that are identified at different scales. Intuitively, the opacity in a particular direction $\Omega$ of such components, composed themselves of sub-components (such as leaves or smaller branching systems) with defined shapes is controlled by two independent factors:

1. On the one hand, it depends on the opacity of the sub-components themselves

2. On the other hand, it depends on the spatial distribution of the subcomponents, and more precisely, on how much the sub-component silhouettes overlap when observed from direction $\Omega$.

In the case of opaque subcomponents (e.g. opaque leaves in a tree crown) the opacity of the composed object in a given direction $\Omega$ is solely a function of the directional overlapping. In the case of porous sub-components, the opacity is the result of these two factors applied to the smaller components. Possibly, the sub-components can themselves be decomposed into smaller components with their own opacity.

In this section, we first briefly recall how the structural organization of a plant can be formalized within a multiscale framework $[15,3]$. We then show how to compute the porosity factors of these elements and use the resulting hierarchical structure to compute light interception. We then show how sensitivity analysis of the model can be carried out to determine the influence of each scale of the hierarchy in the light interception.

2.1. Multiscale representation of plants. Plant 3D mock-ups are represented by sets of geometric components for which the shape, size, spatial co-ordinates and orientation of each component are well defined (Fig. 2.1 a.). These informations can be obtained either from direct measurements $[38,17]$ or from simulation models of plant architecture [35, 12]. In both cases, the multiscale structural information is described as a Multiscale Tree Graph (MTG) [15]. At each scale $i(i=1 \ldots n)$, the plant is regarded as a set of botanical components (e.g. branches, shoots, leaves) arranged as a rooted tree-graph. Components at a scale $i$ are made of components at scale $i+1$ and together define a partition of the set of components at scale $i+1$. Scales 1 corresponds to the whole tree and scale $n$ to the set of leaves (a MTG includes at least these two scales), (Fig. 2.1 d.) Each component is associated with a shape. At the leaf scale $(i=n)$, components (leaves) are represented with a set of polygons. At the other scales $(i \neq n)$, component shapes are convex hulls containing the shapes of their subcomponents (Fig. 2.1).

2.2. Multiscale model of light interception. Using this framework, for each component $j$ at any scale $i$, both $S T A R_{i, j}$ and $P L A_{i, j}$ values can be derived from equations 2.1 and 2.2 :

$$
\begin{gathered}
S T A R_{i, j}=\frac{P L A_{i, j}}{T L A_{i, j}}, \\
P L A_{i, j}=P E A_{i, j} \cdot p_{i, j},
\end{gathered}
$$




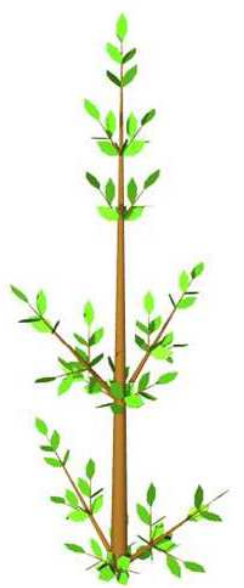

a.

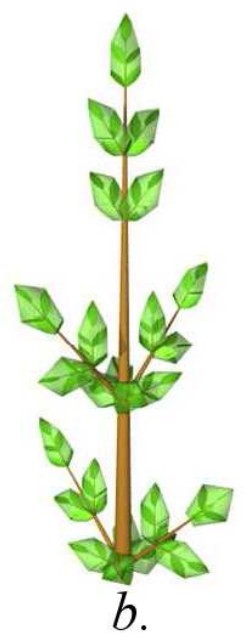

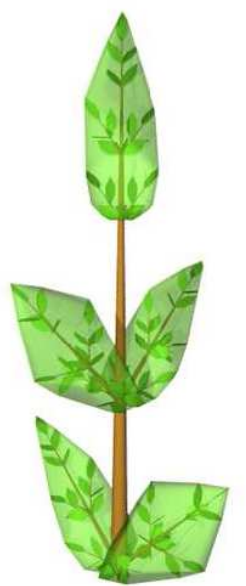

C.

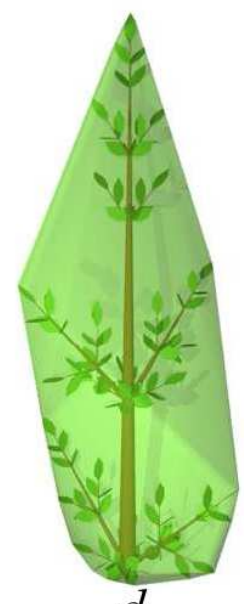

d.

FIG. 2.1. 4 scales decomposition of an artificial tree. Leaves are represented by a set of geometric models (a.). Leafy modules are defined here by botanical branching order 2 (b.), 1 (c.) and $O(d$.$) . Illustration from [3].$

where $P E A_{i, j}$ is the projected envelope area of the component $j$ at scale $i$ and $p_{i, j}$ is its envelope opacity. For leaves (i.e. components at scale $n$ ), $P L A_{n, j}$ and $S T A R_{n, j}$ are simply the projected area of the leaf and the extinction coefficient of the leaf, respectively [36]. At other scales $i(i \neq n)$, components $j$ are porous objects containing subcomponents at scale $i+1$.

In the sequel, we shall show how such a multiscale organization of plants can be used to compute recursively the opacity of a plant crown and to interpret the light interception properties of the plant at different scales.

At each scale $i$ and for each component $j$ at this scale, the envelop opacity $p_{i, j}$ can be estimated by casting a set of regularly spaced beams in the envelope. Let $\mathcal{B}_{i, j}$ be the set of beams intersecting with component $j$ at scale $i$. The origin of beams $b$ ( $b=$ $\left.1 \ldots B_{i, j}\right)$ can be obtained from the cell centers of a regular grid perpendicular to the direction $\Omega$ and positioned above the tree. Each beam is affected with a cross-section area, $A_{b}$, corresponding to the surface area of a grid unit element. Consequently we can associate a volume $\mathcal{V}_{i, j}^{b}$ with each beam $b$

$$
\mathcal{V}_{i, j}^{b}=\mathcal{L}_{i, j}^{b} . A_{b},
$$

where $\mathcal{L}_{i, j}^{b}$ is the travelling distance of beam $b$ in component $j$. Based on the beam sampling we can define estimators $\widehat{\mathcal{V}}_{i, j}$ and $\widehat{P E A}_{i, j}$ of the volume and projected area of the envelope of component $j$ at scale $i$

$$
\widehat{\mathcal{V}}_{i, j} \triangleq \sum_{b=1}^{B_{i, j}} \mathcal{V}_{i, j}^{b} \quad \text { and } \quad \widehat{P E A}_{i, j} \triangleq \sum_{b=1}^{B_{i, j}} P E A_{i, j}^{b}=B_{i, j} \cdot A_{b} .
$$

The beam sampling must be dense enough to verify that $\widehat{\mathcal{V}}_{i, j}$ and $\widehat{P E A}_{i, j}$ provide good estimates of $\mathcal{V}_{i, j}$ and $P E A_{i, j}$. With such a beam sampling, an estimator of the 


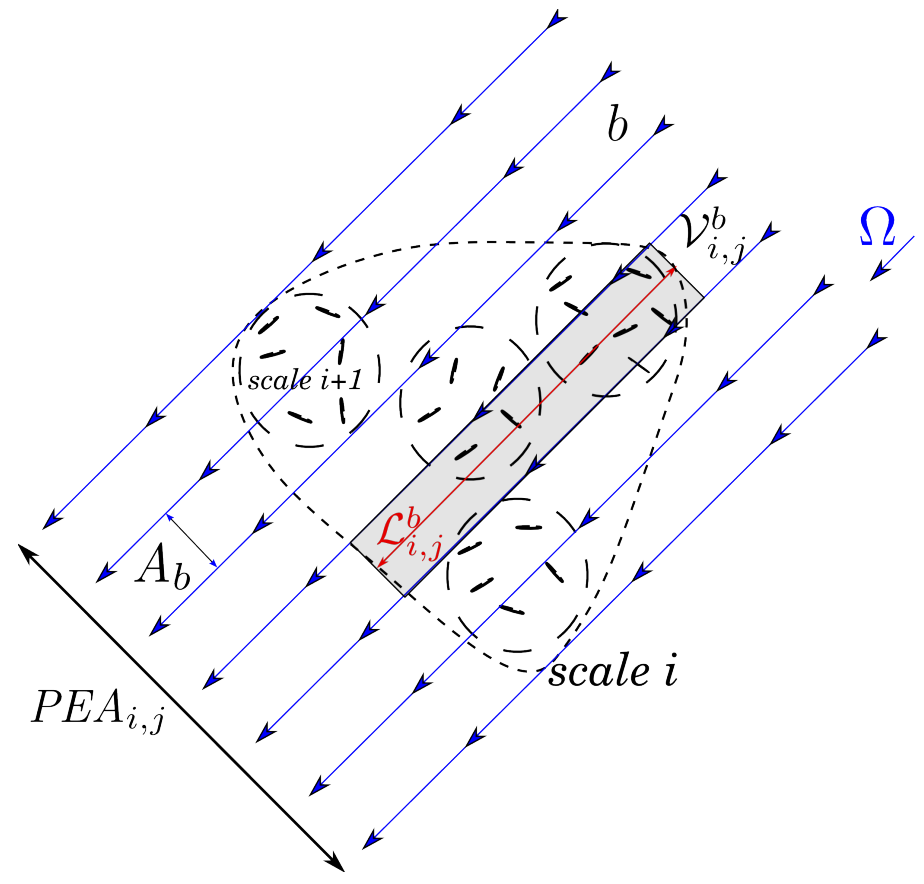

FIG. 2.2. Beam sampling illustration using a 3-scale component representation. A component $j$ at scale $i$ is discretized using a $\Omega$ oriented beam sampling where each beam b has the same cross-section area, $A_{b}$, but a specific length in its envelope, $\mathcal{L}_{i, b}^{b}$, and therefore a specific volume, $\mathcal{V}_{i, j}^{b}$. PEA $A_{i, j}$ is the projected envelope area onto a plane orthogonal to the direction $\Omega$.

envelope opacity, $p_{i, j}$, can be defined as the mean of beam opacities $p_{i, j}^{b}$

$$
\widehat{p}_{i, j} \triangleq \frac{1}{B_{i, j}} \sum_{b=1}^{B_{i, j}} p_{i, j}^{b} .
$$

At any scale $(i<n)$, the model includes two options for computing opacity of a component. The first one (called option A) takes into account the Actual geometry of sub-components in the component envelope. The second one (option $\mathrm{R}$ ) uses the turbid medium analogy, i.e. assumes subcomponents to be Randomly distributed in the envelope volume with uniform density.

Opacity computation in option A. The actual geometry of each subcomponent $k$ in any envelope $j$ at scale $i$ is taken into account. For each beam of the grid positioned above the tree crown, the opacity $p_{i+1, k}^{b}$ of subcomponent $k$, defined as its light interception probability, can be computed from the intersection between the beam and the subcomponent. If the subcomponent is intersected by the beam, i.e. $p_{i+1, k}^{b} \geqslant 0$, the value of $p_{i+1, k}^{b}$ is 1 for a solid object, e.g. a leaf, and lesser than 1 for a porous object. If the subcomponent $k$ is not intersected by the beam $p_{i+1, k}^{b}=0$, therefore the contribution $p_{i, j}^{b}$ of beam $b$ to the opacity of envelope $j$ is computed by taking into account all subcomponents $k$, of scale $i+1$, included in $j$

$$
p_{i, j}^{b}=1-\prod_{k=1}^{n_{j}}\left[1-p_{i+1, k}^{b}\right]
$$

Note that the product is due to the sequence of subcomponents intersected by the beam $b$ but the restriction to these particular subcomponents is made through the value of their beam opacities. Indeed, the above equation shows that $p_{i, j}^{b}$ equals 0 if 
all $p_{i+1, k}^{b}$ are equal to 0 , and $p_{i, j}^{b}$ equals 1 as soon as a subcomponent $k$ intersected by beam $b$ is opaque, $p_{i+1, k}^{b}=1$. For instance, the latter happens when subcomponents are leaves, i.e. $i+1=n$. When $i+1 \neq n$ the value of $p_{i+1, k}^{b}$ is in $[0,1]$ depending on the options of finer scales, see Fig. 2.3.

Opacity computation in option $R$. In option $\mathrm{R}$, subcomponents are randomly and uniformly distributed. The probability that the beam $b$ is not intercepted by the component $j$ at scale $i$ is computed from the product of gap fractions produced by each subcomponent $k$, of scale $i+1$, included in $j$ [39]. The gap fraction for a beam $b$ due to the subcomponent $k$ at scale $i+1$ is defined as the fraction of $A_{b}$ free of the subcomponent projection, i.e.

$$
\frac{A_{b}-P L A_{i+1, k}^{b}}{A_{b}}
$$

where $P L A_{i+1, k}^{b}$ is the portion of component $k$ area projected onto the beam crosssection area, i.e. the restriction of $P L A_{i+1, k}$ to the beam $b$. The probability of no interception, assuming independence between events, is

$$
p_{0 i, j}^{b}=\prod_{k=1}^{n_{j}}\left[1-\frac{P L A_{i+1, k}^{b}}{A_{b}}\right] .
$$

In Eq.(2.9), the product expresses the effect of the uniform random distribution of leaves on the beam opacity in envelope $j$. Moreover, one can see that $\frac{P L A_{i+1, k}^{b}}{A_{b}}$ is exactly the probability of beam interception by component $k$

$$
p_{i+1, k}^{b}=\frac{P L A_{i+1, k}^{b}}{A_{b}},
$$

this leads to

$$
p_{i, j}^{b}=1-p_{0 i, j}^{b}=1-\prod_{k=1}^{n_{j}}\left[1-p_{i+1, k}^{b}\right]
$$

Eq.(2.11) is exactly the same as Eq.(2.8) found in the case of actual distributions of components $k$ in the envelope $j$. However, they differ by the interpretation of the product : in option A, the product expresses the sequence of components $k$ intersected by the beam $b$, while in option $\mathrm{R}$, it expresses the random position of components. This analogy between both equations is further investigated in section 5.3.

In option $\mathrm{R}$, we thus need to compute $P L A_{i+1, k}^{b}$ for each beam. To carry out this computation, we use the assumption of uniform density of foliage which make it possible to write

$$
\frac{P L A_{i+1, k}^{b}}{\mathcal{V}_{i, j}^{b}}=\frac{P L A_{i+1, k}}{\mathcal{V}_{i, j}}
$$

as $\mathcal{V}_{i, j}^{b}=A_{b} \mathcal{L}_{i, j}^{b}$, Eq.(2.10) and (2.12) can be combined to express opacity $p_{i+1, k}^{b}$

$$
p_{i+1, k}^{b}=\frac{P L A_{i+1, k} \mathcal{V}_{i, j}^{b}}{A_{b} \mathcal{V}_{i, j}}=\frac{P E A_{i+1, k} p_{i+1, k} \mathcal{L}_{i, j}^{b}}{\mathcal{V}_{i, j}},
$$




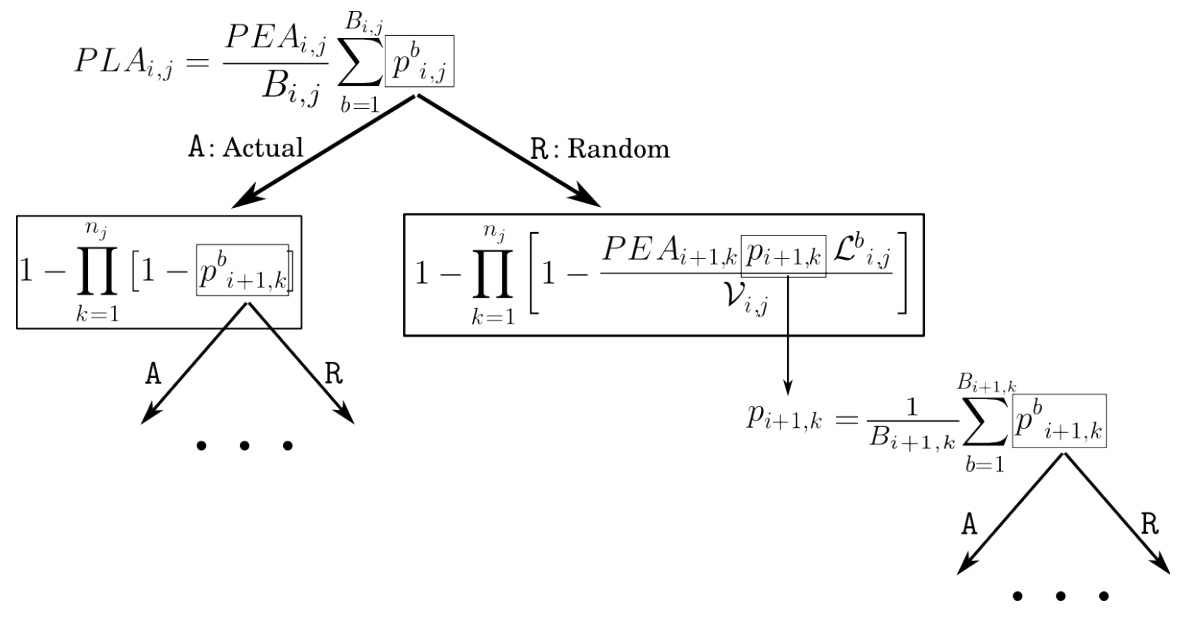

FIG. 2.3. Recursive pattern for computing the PLA of an element through its envelope opacity. The combined options of inter-scale distribution determine the path to follow in this equation scheme. The recursion ends when last scale is reached, this scale must have a known or fixed opacity. For instance in this study leaf are considered opaque, i.e. opacity $=1$. Hence for all-A scenario the value of every $p_{i, j}^{b}$ is either 0 or 1 , for all $i$ and $j$.

Eq.(2.11) can then be written

$$
p_{i, j}^{b}=1-\prod_{k=1}^{n_{j}}\left[1-\frac{P E A_{i+1, k} p_{i+1, k} \mathcal{L}_{i, j}^{b}}{\mathcal{V}_{i, j}}\right] .
$$

To summarize, we showed that the opacity of component $j$ at scale $i$ can be expressed by a unique set of recursive equations that enables us to express $P L A_{i, j}$ for each $(i, j)$ as

$$
P L A_{i, j}=P E A_{i, j} \frac{1}{B_{i, j}} \sum_{b=1}^{B_{i, j}} p_{i, j}^{b} .
$$

This makes it possible to evaluate $S T A R_{i, j}$ from Eq.(2.3) based on an estimate of the plant total leaf area [45, 32]. The scheme described in Fig. 2.3 illustrates the recursive procedure that uses Eq.(2.8) or Eq.(2.14) depending on the scenario. It can be used with any number of scales and either option A or R can be independently used at each scale.

2.3. Assessing light interception. Using this modeling framework, any particular scale can be added, removed or its spatial distribution switched from one option to another, e.g. changing the actual position of leaves (option A) to the hypothesis of random distribution of leaf area density (option R). This will be used to analyze the influence of one specific scale in light interception. In case of a crown filled with leaves, i.e. the number of scales is two, option A corresponds to the actual leaf distribution within the crown, e.g. as digitized in the field. Otherwise, option $\mathrm{R}$ leads to the basic random distributionof leaf area in the tree crown, as used in many turbid medium models $[8,22]$. In case of more scales, scenario corresponding to the selected combination of options $A$ and $R$ is encoded as a 6string of characters, containing number of scales minus 1 characters. The first and last characters of the 
string refer to scale 2 and scale $n$, respectively. The option for scale 1 can only be A, i.e. the actual tree crown. This is why scale 1 is not included in the string. For example, let us consider a tree organized in three scales, e.g. crown, shoots and leaves. A scenario AA would correspond to actual arrangement of shoots within the crown and to actual arrangement of leaves within shoots. This corresponds to the real plant structure and is assumed to be the true value. Another example illustrated in Fig. 2.4 is the scenario RR corresponding to the Oker-Blom and Kellomaki's model [30] where leaves and shoots are randomly distributed in shoots and in the crown, respectively. In addition, any inner scale $(i \in[2, n-1])$ can be discarded to distribute components at scale $i$ directly in envelopes at scale $i-2$. Discarded scales are denoted "-" in the scenario string. For instance, in the example above, the scenario $-\mathrm{R}$ means that leaves are directly randomly distributed in the tree crown. Finally, note that discarding a scale and then using the actual position is equivalent to use the actual distribution for the discarded scale, i.e. $-\mathrm{A} \equiv \mathrm{AA}$.

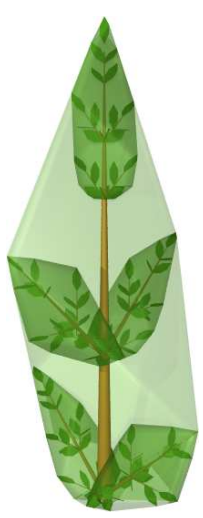

$\mathrm{AA}$

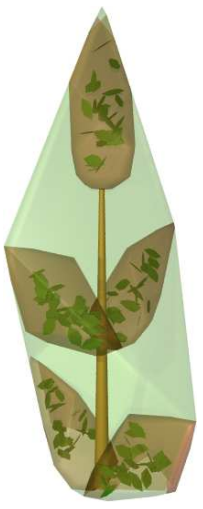

AR

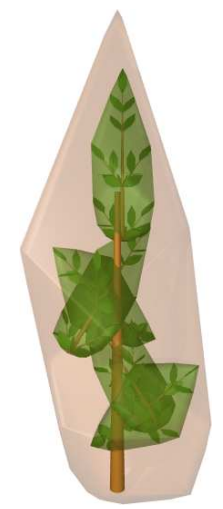

RA

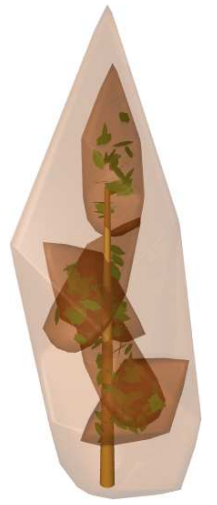

$\mathrm{RR}$

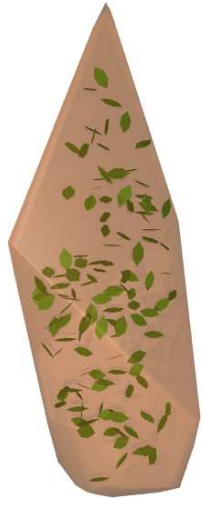

$-\mathrm{R}$

FIG. 2.4. Example of distribution options for an object with 3 scales, namely crown, shoot and leaves. Hence a 2-character string represents inter-scale distributions options. AA represents the actual distribution of leaves in the crown. With AR the actual position of shoots are used but the opacity of their envelopes are computed with the turbid medium analogy whereas RA uses the turbid medium analogy for shoots but with their real opacity taken into account. The RR distribution corresponds to the model where leaves and shoots are randomly distributed in shoots and in the crown, respectively, i.e. grouping model. Finally, $-R$ uses the turbid medium analogy and supposes a uniform distribution of leaves in the crown.

As presented in section 2.2, our goal is to estimate for each plant its global light interception efficiency in terms of $S T A R$. This efficiency is evaluated at plant scale, i.e. $i=1$ and $j=1$, by integrating directional $S T A R$ values over the sky directions $\Omega$. In the following $i$ and $j$ indexed notations will thus be omitted and the $\Omega$ indexed notation will indicate directional quantities whereas $\Omega$-free notation will stand for integrated values. $\Omega$-integrated values for $S T A R$ and $P E A$ are obtained by summing up the directional values weighted by coefficients, $\omega_{\Omega}$, derived from the Standard OverCast sky radiance distribution [37]

$$
S T A R \triangleq \sum_{\Omega} S T A R_{\Omega} \cdot \omega_{\Omega} \quad \text { and } \quad P E A \triangleq \sum_{\Omega} P E A_{\Omega} \cdot \omega_{\Omega} .
$$

$S T A R_{\Omega}$ values are computed according to Eq.(2.1) from $P L A_{\Omega}$ whose evaluation is described in Fig. 2.3. Originally the leaf dispersion parameter, $\mu$, introduced in Beer- 
Lambert equation as a $L A D$ modifier, expresses the departure of the actual crown gap-fraction from the gap-fraction of a crown with random distribution of leaves and equivalent leaf area density. Indeed, $\mu$ is 1 for random distribution of leaves whereas leaf clumping leading to higher crown porosity yields a $\mu$ strictly lesser than 1 . It can be extended to compare two scenarii, expressing a relative dispersion coefficient, $\mu$, of a test porosity, $p_{0}$, against a reference one, $p_{0<r e f>}$. By analogy with the BeerLambert formalism where $p_{0<r e f>}>$ would be expressed using Eq.(1.1) and $p_{0}$ using Eq.(1.2) the following relationship is defined [40]

$$
p_{0}=p_{0<r e f>}{ }^{\mu} .
$$

Using the complementary relationship between opacity and porosity and equations (2.1) and (2.2), an $\Omega$-integrated $\mu$ value can be expressed as a function of $S T A R$ and $P E A$

$$
\mu=\frac{\log \left(1-\frac{S T A R \cdot T L A}{P E A}\right)}{\log \left(1-\frac{S T A R_{<\text {ref }>. T L A}}{P E A}\right)} .
$$

It allows us studying the global or scale by scale spatial organization through the computation of $\mu$ from specific scenario. For instance, in the example illustrated in Fig. 2.4, the definition of $\mu$ as introduced by Nilson [27] corresponds to comparing the situation $\mathrm{AA}$ to the reference situation $-\mathrm{R}$ and is therefore noted $\mu(\mathrm{AA} /-\mathrm{R})$. The $S T A R$ of these scenarii are noted $S T A R_{<\mathrm{AA}>}$ and $S T A R_{<-\mathrm{R}>}$, respectively. The scale by scale organization is given by scenarii which differ by only one letter corresponding to the studied scale. For instance the shoot distribution within the crown and the leaves distribution in the shoots are given by the ratio of $S T A R_{<\mathrm{AA}>}$ to $S T A R_{<\mathrm{RA}>}$ and $S T A R_{<\mathrm{AA}>}$ to $S T A R_{<\mathrm{AR}>}$, respectively.

\section{Plant database.}

3.1. Plant material. Four 4-year old peach trees (variety August Red) were digitized one month after bud break in May 2001, in CTIFL-Balandran in SouthEast of France $\left(43^{\circ} 83^{\prime} \mathrm{N}, 4^{\circ} 35^{\prime} \mathrm{E}\right)$. Two of them were trained as tight goblet (TG) system, and the two other ones as wide double-Y (WDY). TG is an open center (goblet-shaped) structure employing several primary scaffolds. Planting distances in TG system were $6 \times 3 \mathrm{~m}$. WDY derives from goblet, with larger planting distances, $7 \times 4 m$, and four primary scaffolds arranged by pairs [14]. Tree height was about $2.5 m$.

Four 3-year old mango trees grown in a commercial farm were digitized in March 2001 in Saint-Paul, La Réunion Island $\left(20^{\circ} 53^{\prime} \mathrm{S}, 55^{\circ} 32^{\prime} \mathrm{E}\right)$. Two of them belonged to variety Lirfa, and the two other ones to variety José. A square planting system was used with a 6-meter distance between trees and a north-west-south-east row orientation. Tree height was about $1.5 \mathrm{~m}$. Full information on peach and mango trees can be found in [42] and [44], respectively.

3.2. Three-dimensional plant digitizing and reconstruction. All trees were 3D-digitized with a 3Space Fastrak electro-magnetic device (Polhemus inc., Colchester, VT, USA). The digitizer includes a magnetic field generator and a pointer to be positioned by an operator at each point to be measured. The pointer includes coils where electrical currents are induced when located in the magnetic fields. The 

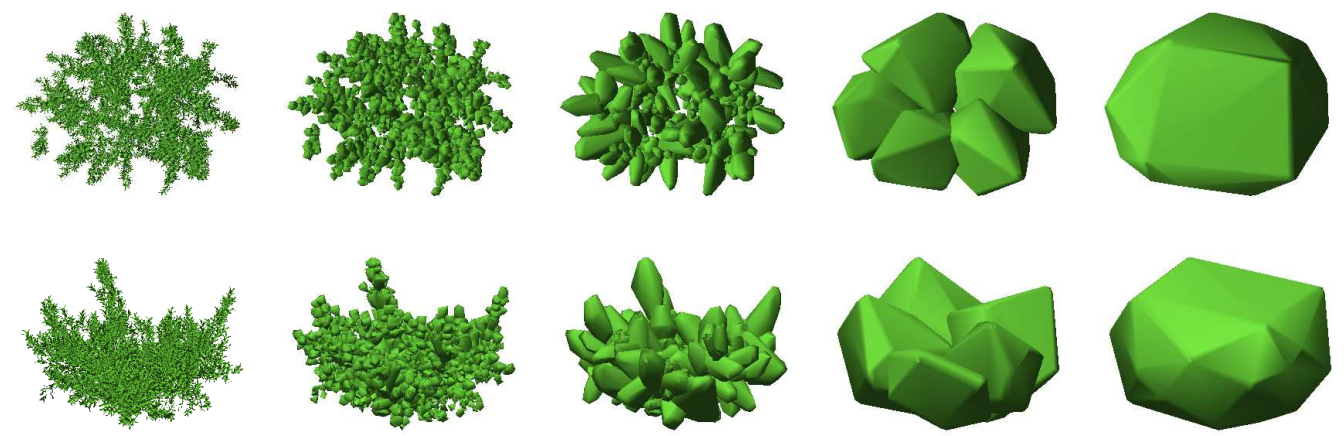

Fig. 3.1. Peach tree scale decomposition. Top : top view, bottom : side view and from left to right : leaves, CYS, OYOS, Scaffold, Crown
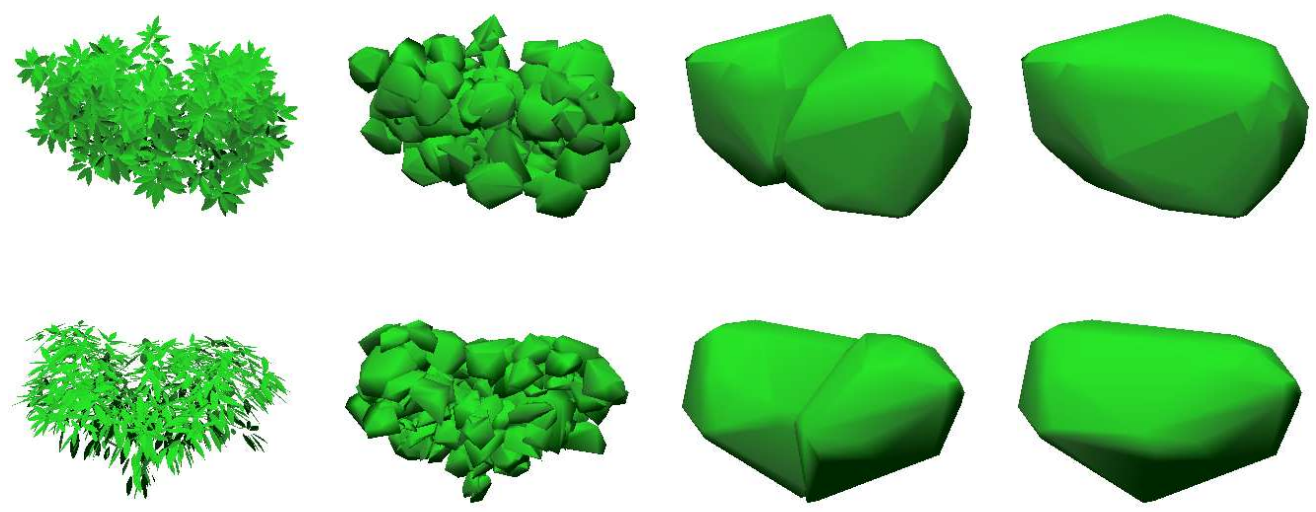

FIG. 3.2. Mango tree scale decomposition. Top : top view, bottom : side view and from left to right : leaves, CYS, Scaffold, Crown

spatial co-ordinates and orientation angles in 3D can be derived from the values of induced currents [33]. Plant digitizing was driven by software Pol95 [1].

Mango trees were digitized at leaf scale according to Sinoquet et al.'s [41] method. For each leaf, the pointer was located at the junction between lamina and petiole, with pointer $X$-axis parallel to the midrib and pointer $X-Y$ plane parallel to lamina. With this configuration, measured angles were midrib azimuth, midrib elevation and rolling angle of lamina around the midrib. In addition, leaf length was measured with a ruler. Fifty leaves were harvested for leaf area $\left(A \mathrm{~cm}^{2}\right)$ measurement with a planimeter, and leaf length $(L \mathrm{~cm})$ was measured with a ruler. This sample was used to set a relationship between $A$ and $L^{2}$ :

$$
A=k \cdot L^{2} .
$$

Coefficient $k$ was constant for both varieties, with a value of 0.1826 . Peach trees were digitized at the leafy shoot scale according to Sinoquet and Rivet's method 
[38]. For each leafy shoot, the spatial bottom and top co-ordinates of the shoot were recorded with the digitizer. For each shoot type in each cultivar, namely short and long $(>5 \mathrm{~cm})$ shoots, 15 to 30 shoots were digitized at leaf scale, as described above. These data were used to establish foliage reconstruction rules for all digitized shoots. Reconstruction rules included allometric relationships at shoot and leaf scale, random sampling in leaf angle distributions and additional hypotheses. The reconstruction method has been fully presented and assessed in [42]. At the same time as digitizing, the position of the digitized organ in the multiscale tree organization was recorded using Godin et al.'s method [17]. For mango trees 4 scales were used : plant, scaffold, current-year-shoot (CYS) i.e. leafy shoot, and leaf. One additional scale was used for peach trees, i.e. one-year-old shoot (OYOS) between scaffold and CYS scales. Of course reconstructed peach leaves were assigned to the corresponding digitized shoot.Finally a database was obtained for each tree, as a collection of leaves explicitly distributed in 3D space and related to the multiscale organization of the tree.

TABLE 3.1

Scale by scale component demography and their envelope volume, contained leaf area and LAD. Abbreviations : $O Y O S=$ One Year Old Shoot; CYS $=$ Current Year Shoot; LAD $=$ Leaf Area Density.

\begin{tabular}{|c|c|c|c|c|c|}
\hline & Scale & $\mathrm{Nb}$ Elmt & Volume $\left(d m^{3}\right)$ & Leaf Area $\left(d m^{2}\right)$ & $\operatorname{LAD}\left(d m^{-1}\right)$ \\
\hline \multirow{5}{*}{ 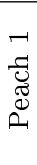 } & Crown & 1 & 11811.50 & 2773.85 & 0.24 \\
\hline & Scaffold & 4 & $2832.70 \pm 1214.52$ & $693.45 \pm 232.08$ & $0.253 \pm 0.04$ \\
\hline & OYOS & 86 & $14.02 \pm 29.27$ & $9.10 \pm 14.85$ & $1.31 \pm 0.83$ \\
\hline & CYS & 1966 & $0.97 \pm 1.54$ & $1.41 \pm 1.95$ & $1.90 \pm 0.58$ \\
\hline & Leaf & 14405 & - & $0.19 \pm 0.05$ & - \\
\hline \multirow{5}{*}{ 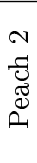 } & Crown & 1 & 12200.89 & 2889.69 & 0.24 \\
\hline & Scaffold & 5 & $1915.22 \pm 507.09$ & $577.74 \pm 190.48$ & $0.30 \pm 0.04$ \\
\hline & OYOS & 88 & $13.98 \pm 22.88$ & $9.99 \pm 12.51$ & $1.22 \pm 0.72$ \\
\hline & CYS & 1522 & $1.40 \pm 2.06$ & $1.90 \pm 2.32$ & $1.77 \pm 0.53$ \\
\hline & Leaf & 13709 & - & $0.21 \pm 0.55$ & - \\
\hline \multirow{5}{*}{ 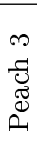 } & Crown & 1 & 12442.29 & 3242.96 & 0.26 \\
\hline & Scaffold & 5 & $1934.32 \pm 935.55$ & $648.58 \pm 266.30$ & $0.34 \pm 0.05$ \\
\hline & OYOS & 93 & $15.51 \pm 24.89$ & $11.30 \pm 15.24$ & $1.35 \pm 1.00$ \\
\hline & CYS & 1583 & $1.52 \pm 2.30$ & $2.05 \pm 2.78$ & $1.77 \pm 0.52$ \\
\hline & Leaf & 15118 & - & $0.21 \pm 0.55$ & - \\
\hline \multirow{5}{*}{ 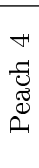 } & Crown & 1 & 15911.77 & 3144.29 & 0.20 \\
\hline & Scaffold & 6 & $2060.11 \pm 773.34$ & $524.04 \pm 186.99$ & $0.26 \pm 0.04$ \\
\hline & OYOS & 101 & $12.48 \pm 20.19$ & $8.30 \pm 9.99$ & $1.39 \pm 1.10$ \\
\hline & CYS & 1990 & $1.17 \pm 1.91$ & $1.58 \pm 2.01$ & $1.84 \pm 0.57$ \\
\hline & Leaf & 15589 & - & $0.20 \pm 0.06$ & - \\
\hline \multirow{5}{*}{ 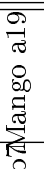 } & Crown & 1 & 1146.66 & 631.64 & 0.55 \\
\hline & Scaffold & 3 & $417.03 \pm 68.42$ & $210.54 \pm 11.95$ & $0.51 \pm 0.07$ \\
\hline & CYS & 139 & $6.07 \pm 4.81$ & $4.54 \pm 2.64$ & $1.06 \pm 0.72$ \\
\hline & Leaf & 1475 & - & $0.43 \pm 0.17$ & - \\
\hline & Crown & 1 & 2073.24 & 1221.87 & 0.59 \\
\hline \multirow{3}{*}{$\begin{array}{l}\infty \\
\infty \\
\stackrel{\infty}{\Xi} \\
\stackrel{\Xi}{\Sigma}\end{array}$} & Scaffold & 3 & $793.35 \pm 229.78$ & $407.29 \pm 126.59$ & $0.51 \pm 0.02$ \\
\hline & CYS & 252 & $9.00 \pm 6.47$ & $4.85 \pm 2.70$ & $0.73 \pm 0.52$ \\
\hline & Leaf & 2759 & - & $0.44 \pm 0.19$ & - \\
\hline \multirow{4}{*}{ 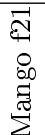 } & Crown & 1 & 1188.56 & 509.19 & 0.43 \\
\hline & Scaffold & 2 & $614.09 \pm 93.89$ & $254.60 \pm 55.84$ & $0.41 \pm 0.03$ \\
\hline & CYS & 147 & $4.80 \pm 4.93$ & $3.46 \pm 2.40$ & $0.77 \pm 0.48$ \\
\hline & Leaf & 1367 & - & $0.37 \pm 0.18$ & - \\
\hline 80 & Crown & 1 & 869.39 & 492.87 & 0.57 \\
\hline \multirow{3}{*}{ 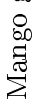 } & Scaffold & 7 & $146.10 \pm 68.07$ & $70.41 \pm 32.48$ & $0.48 \pm 0.05$ \\
\hline & CYS & 99 & $8.27 \pm 6.92$ & $4.98 \pm 3.19$ & $0.83 \pm 0.50$ \\
\hline & Leaf & 1135 & - & $0.43 \pm 0.19$ & - \\
\hline
\end{tabular}


4. Results and clumping analysis. $\Omega$-integrated values for $S T A R$ and $\mu$ according to several hypothesis are shown in Tab. 4.1 and Tab. 4.2 for peach and mango trees respectively. Actual $S T A R$ values (line 0 ) for peach trees did not show a large range i.e. between 0.245 and 0.275 . This means that foliage irradiance was about $25 \%$ of the available light above the tree. For mango trees the range of $S T A R$ values was greater, i.e. between 0.243 and 0.364 . These values are in the interval commonly reported for fruit trees [47]. For both species, the classical assumption of random distribution of leaves in crowns (row 1) led to large over-estimation of the STAR values. Therefore light interception models based on this assumption are biased as already reported by $[46,9,11]$. This confirms that the actual foliage distribution shows significant departure from randomness. The values of the dispersion parameter $\mu$ were between 0.55 and 0.61 and between 0.70 and 0.81 for peach and mango trees respectively, expressing a clumped organization of the foliage and higher clumping for peach trees than for mango trees.

The full grouping model (all-R, row 2) underestimated light interception (for all trees, but Peach1). Indeed $\mu$ values above 1 means that the leaves in the actual canopy were less overlapping than they would have been in the canopy generated from the full grouping model. Consequently actual $S T A R$ values were comprised between these two most usual modeling approaches. However the two species showed different behavior : actual STAR of peach trees was overestimated by $30 \%$ with scenario - --R whereas scenario all-R only underestimated actual $S T A R$ by $10 \%$. In case of mango trees, the overestimation given by scenario -- $\mathrm{R}$ was around $12 \%$ and equal to the underestimation given by scenario all $-\mathrm{R}$.

A deeper analysis was made by comparing the actual $S T A R$ values to those obtained by switching one scale option from A to R. This allows studying the dispersion pattern at the switched scale. For instance, the leaf dispersion within CYS in peach trees was assessed by comparing scenario AAAA to scenario AAAR. For peach trees (Tab.4.1), only CYS in OYOS (row 4) showed a clear random dispersion $(\mu \simeq 1$ ). $S T A R_{<\mathrm{AAAR}>}$ and $S T A R_{<\mathrm{RAAA}>}$ (row 3 and 6 respectively) were slightly lesser than the actual value, thus the distribution of leaves in CYS and scaffolds in crowns was slightly regular $\left(\mu \simeq 1.11\right.$ and $\mu \simeq 1.15$ respectively). Finally $S T A R_{<\text {ARAA }>}$ (row 5) was a bit greater than the actual value, showing a small clumping trend of OYOS in scaffolds $(\mu \simeq 0.91$ ). For mango trees (Tab.4.2), scenario AAR and ARA (row 3 and 4 ) led to small under and overestimation of actual $S T A R$ expressing slight regularity and clumpiness of leaves in CYS and CYS in scaffolds, respectively. By contrast, scenario RAA (row 5) markedly underestimated actual STAR meaning that the actual distribution of scaffolds in the crown was regular $(\mu \simeq 1.26)$. The dispersion pattern for each scale was also assessed by comparing the $S T A R$ behavior values of full grouping scenario $($ all $-\mathrm{R})$ to those obtained by switching one scale option from $\mathrm{R}$ to $\mathrm{A}$ (row 7 to 10 in Tab.4.1 and row 6 to 8 in Tab.4.2 for peach and mango trees respectively). This analysis confirmed the dispersion previously found at each scale.

As scaffold scale was shown to be regular, we tested a scenario where this scale was discarded (row 11 and 9 for peach and mango trees, respectively). Theoretically this should lead to $S T A R$ values closer to the actual ones because the scaffold subcomponents are no more confined within the scaffold envelopes but distributed in the full crown volume. It is expected to be a way to simulate the regularity of scaffolds 
TABLE 4.1

$\Omega$-integrated STAR for Peach trees.

\begin{tabular}{|c|c|c|c|c|c|}
\hline & & Peach1 & Peach2 & Peach 3 & Peach 4 \\
\hline 0 & AAAA & 0.2477 & 0.2715 & 0.2456 & 0.2754 \\
\hline \multirow{2}{*}{1} & $---R$ & 0.3389 & 0.3483 & 0.3239 & 0.3591 \\
\hline & $\mu(\mathrm{AAAA} /---\mathrm{R})$ & 0.5482 & 0.6127 & 0.5734 & 0.6082 \\
\hline \multirow{2}{*}{2} & RRRR & 0.2422 & 0.2347 & 0.2177 & 0.2504 \\
\hline & $\mu(\mathrm{AAAA} / \mathrm{RRRR})$ & 1.0368 & 1.2582 & 1.2110 & 1.1599 \\
\hline \multirow{2}{*}{3} & AAAR & 0.2372 & 0.2497 & 0.2312 & 0.2542 \\
\hline & $\mu(\mathrm{AAAA} / \mathrm{AAAR})$ & 1.071 & 1.1445 & 1.1036 & 1.1331 \\
\hline \multirow{2}{*}{4} & AARA & 0.2480 & 0.2697 & 0.2442 & 0.2719 \\
\hline & $\mu(\mathrm{AAAA} / \mathrm{AARA})$ & 0.9987 & 1.01 & 1.0096 & 1.021 \\
\hline \multirow{2}{*}{5} & ARAA & 0.2594 & 0.2890 & 0.2586 & 0.2927 \\
\hline & $\mu(\mathrm{AAAA} / \mathrm{ARAA})$ & 0.9281 & 0.8971 & 0.9154 & 0.904 \\
\hline \multirow{2}{*}{6} & RAAA & 0.2349 & 0.2412 & 0.2226 & 0.2585 \\
\hline & $\mu(\mathrm{AAAA} / \mathrm{RAAA})$ & 1.0868 & 1.2071 & 1.1782 & 1.1257 \\
\hline \multirow{2}{*}{7} & RRRA & 0.2518 & 0.2519 & 0.2300 & 0.2676 \\
\hline & $\mu$ (RRRA/RRRR) & 1.0643 & 1.1145 & 1.0884 & 1.1082 \\
\hline \multirow{2}{*}{8} & RRAR & 0.2421 & 0.2352 & 0.2184 & 0.2514 \\
\hline & $\mu$ (RRAR/RRRR) & 0.9995 & 1.003 & 1.0048 & 1.0066 \\
\hline \multirow{2}{*}{9} & RARR & 0.2261 & 0.2235 & 0.2100 & 0.2379 \\
\hline & $\mu$ (RARR/RRRR) & 0.8999 & 0.9304 & 0.9466 & 0.9274 \\
\hline \multirow{2}{*}{10} & ARRR & 0.2487 & 0.2650 & 0.2416 & 0.2703 \\
\hline & $\mu(\mathrm{ARRR} / \mathrm{RRRR})$ & 1.0432 & 1.2087 & 1.1782 & 1.1257 \\
\hline \multirow{2}{*}{11} & -RRR & 0.2803 & 0.2789 & 0.2639 & 0.3078 \\
\hline & $\mu(\mathrm{AAAA} /-\mathrm{RRR})$ & 0.8117 & 0.9554 & 0.8835 & 0.8280 \\
\hline \multirow{2}{*}{12} & $--R R$ & 0.3061 & 0.3035 & 0.2898 & 0.3221 \\
\hline & $\mu(\mathrm{AAAA} /--\mathrm{RR})$ & 0.6871 & 0.8202 & 0.7386 & 0.7615 \\
\hline \multirow{2}{*}{13} & $\mathrm{AA}-\mathrm{R}$ & 0.2522 & 0.2578 & 0.2443 & 0.2651 \\
\hline & $\mu(\mathrm{AAAA} / \mathrm{AA}-\mathrm{R})$ & 0.9717 & 1.0882 & 1.0094 & 1.0627 \\
\hline
\end{tabular}

distribution in crown. Indeed $S T A R$ values in scenario -RRR and -RR were greater than values given by the full grouping model for peach and mango trees, respectively. They were also greater than $S T A R$ values given by scenario ARRR and ARR, meaning that disregarding the scaffold scale led to overestimate the actual regular dispersion of scaffolds. For all trees the actual $S T A R$ value was in between the values given by full grouping scenario and that disregarding the scaffold scale. For peach trees scenario AA-R (row 13) gave STAR values very close to the actual ones. The profit of this scenario will be discussed in the next section.

\section{Discussion.}

5.1. Requirements in canopy structure description for an accurate estimation of light interception. Exhaustive measurement of the canopy structure, e.g. to get data for scenario all-A, is extremely tedious, especially for the peach trees where the number of leaves was about 15,000 per tree. Conversely, the simplest scenario - $R$ only uses a few data to describe the tree structure, namely crown shape and volume and total leaf area. However the computed light interception was shown to be largely overestimated. This confirms that the actual tree foliage distribution in crowns is not uniform and shows high clumping [11]. Consequently, the simulation models using this scenario $[29,7]$ should include a calibration parameter $\mu$. By contrast, the full grouping scenario underestimated light interception for both species. Moreover the Oker-Blom and Kellomaki's [30] model, i.e. --RR and -RR for peach and mango trees, respectively, yielded $S T A R$ values close to the actual ones, but only for mango 
TABLE 4.2

$\Omega$-integrated STAR for Mango trees.

\begin{tabular}{|c|c|c|c|c|c|}
\hline & & Mango a 19 & Mango b7 & Mango f21 & Mango g5 \\
\hline 0 & AAA & 0.2824 & 0.2434 & 0.3637 & 0.3235 \\
\hline \multirow{2}{*}{1} & $--\mathrm{R}$ & 0.3282 & 0.2717 & 0.4025 & 0.3564 \\
\hline & $\mu(\mathrm{AAA} /--\mathrm{R})$ & 0.7039 & 0.7315 & 0.8112 & 0.7982 \\
\hline \multirow{2}{*}{2} & RRR & 0.2544 & 0.2193 & 0.3159 & 0.2811 \\
\hline & $\mu(\mathrm{AAA} / \mathrm{RRR})$ & 1.2157 & 1.2552 & 1.2809 & 1.3077 \\
\hline \multirow{2}{*}{3} & AAR & 0.2736 & 0.2347 & 0.3478 & 0.3084 \\
\hline & $\mu(\mathrm{AAA} / \mathrm{AAR})$ & 1.064 & 1.0886 & 1.0859 & 1.1019 \\
\hline \multirow{2}{*}{4} & ARA & 0.2915 & 0.2497 & 0.3643 & 0.3053 \\
\hline & $\mu(\mathrm{AAA} / \mathrm{ARA})$ & 0.9366 & 0.9391 & 0.9964 & 1.1240 \\
\hline \multirow{2}{*}{5} & RAA & 0.2507 & 0.2124 & 0.3191 & 0.2927 \\
\hline & $\mu(\mathrm{AAA} / \mathrm{RAA})$ & 1.2465 & 1.3346 & 1.2596 & 1.2163 \\
\hline \multirow{2}{*}{6} & RRA & 0.2608 & 0.2222 & 0.3252 & 0.2892 \\
\hline & $\mu(\mathrm{RRA} / \mathrm{RRR})$ & 1.0458 & 1.0267 & 1.0495 & 1.0519 \\
\hline \multirow{2}{*}{7} & RAR & 0.2444 & 0.2065 & 0.3076 & 0.2820 \\
\hline & $\mu(\mathrm{RAR} / \mathrm{RRR})$ & 0.9335 & 0.8922 & 0.9576 & 1.0058 \\
\hline \multirow{2}{*}{8} & ARR & 0.2828 & 0.2456 & 0.3519 & 0.2956 \\
\hline & $\mu(\mathrm{ARR} / \mathrm{RRR})$ & 1.2195 & 1.2830 & 1.2047 & 1.0949 \\
\hline \multirow{2}{*}{9} & $-\mathrm{RR}$ & 0.3018 & 0.2582 & 0.3670 & 0.3262 \\
\hline & $\mu(\mathrm{AAA} /-\mathrm{RR})$ & 0.8683 & 0.8577 & 0.9829 & 0.9825 \\
\hline \multirow{2}{*}{10} & $A-R$ & 0.3068 & 0.2587 & 0.3846 & 0.3190 \\
\hline & $\mu(\mathrm{AAA} / \mathrm{A}-\mathrm{R})$ & 0.8363 & 0.8537 & 0.8949 & 1.0298 \\
\hline
\end{tabular}

trees. As the Oker-Blom and Kellomaki's model can be regarded as a partial grouping model (i.e. leaves randomly distributed in shoots and shoots directly distributed at random in the tree), this questions the number of botanical scales that must be used in grouping models. However the Oker-Blom and Kellomaki's model was reported to be successful for conifer species [30], and our results show that this approach can be also used for some, but not all, fruit tree species. In the case of peach trees, the grouping scenarii did not work satisfactorily, however the scenario AA-R gave nice results. This means that good estimations of light interception can be obtained by measuring the actual position of OYOS in the crown. In practice, this kind of measurement is really tractable [42] as a peach tree usually includes only about one hundred OYOS (see Tab.3.1). Moreover the OYOS scale corresponds to the management unit used to train the tree (i.e. the fruit grower selects a given number of OYOS in the sunlit zones of the tree and prunes the other ones).

Therefore an interesting use of the proposed modeling framework is to find out scenarii of canopy structure description as simple as possible that enables an accurate estimation of light interception without artificially using leaf dispersion parameters $\mu$ (especially because there is about no means to estimate $\mu$ ).

\subsection{Sensitivity analysis.}

Measurement errors effect. Magnetic digitizing is prone to measurement errors both on the location and orientation of organs. The error on spatial co-ordinates is typicaly less than $1 \mathrm{~mm}$ in controlled environment [24] and is less than $1 \mathrm{~cm}$ for field measurements [43]. The impact of such errors on $S T A R$ computation has already been assessed on peach trees by Sonohat et al. [42]. In this case, measurements were conducted at shoot scale and leaf position and shape were reconstructed using allometric rules. It was shown that the $S T A R$ estimation at shoot scale was inacurrate while it was satisfactory at plant scale. In the case of mango trees, which were digitized at leaf scale, we quantified the error in $S T A R$ estimation by generating mock-ups where 
random errors were introduced in leaf spatial co-ordinates and orientation angles. We designed two procedures for this. We first used a uniform distribution in the range $[-\varepsilon, \varepsilon]$ to modify the co-ordinates and the orientation of each leaf. Alternatively, we used Student's distribution that yields a greater proportion of small errors. The impact of these two distributions on STAR computation was evaluated on a set of 100 mock-ups with $\varepsilon$ values defined so that the new position of each leaf is in a sphere of radius $0.1,0.2,0.5,1,2$ and $5 \mathrm{~cm}$ centered on its original position. Orientation angles were modified similarly with $\varepsilon$ ranging from $5^{\circ}$ to $45^{\circ}$. Results showed that positioning errors less than $1 \mathrm{~cm}$ had little effect on light interception capacities at plant scale (Fig. 5.1 a.), and for $1 \mathrm{~cm}$, corresponding to the field measurement error, the STAR value error using scenario all-A was less than $5 \%$ regardless of the distribution used. For measurement errors greater than $1 \mathrm{~cm}$ the foliage rapidly tended to occupy a more important volume leading to a lower leaf area density and thus a greater STAR. This effect was much more marked when errors were generated using a uniform distribution (Fig. 5.1 a.). Note however that errors greater than $1 \mathrm{~cm}$ should be avoided if measurements are carefully conducted. Orientation errors had only neglectible effect on $S T A R$ values integrated over the sky-vault (less than $0.5 \%$ for all $\varepsilon$ values, data not shown).

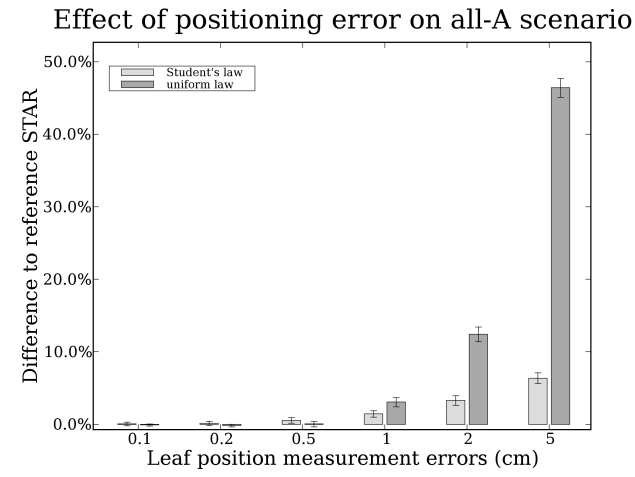

$a$.

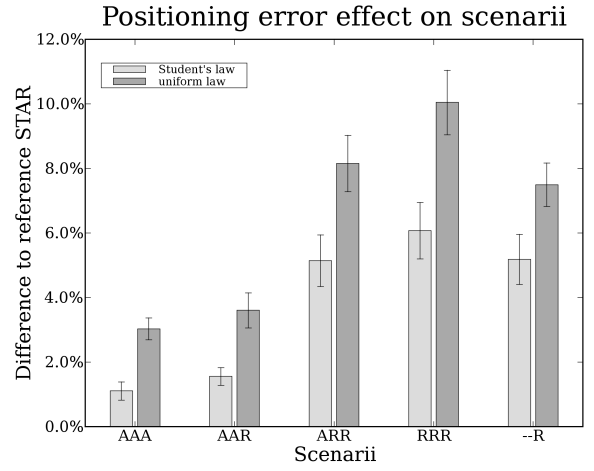

$b$.

FIG. 5.1. Influence of measurement errors on the STAR estimation. a. : effect of increasing measurement error. Random error generated by a uniform distribution (dark grey) or Student's distribution (light grey) is introduced on leaf spatial coordinates so that the new position is in a sphere of radius $0.1,0.2,0.5,1,2$ and $5 \mathrm{~cm}$ centered on its original position. $b . \quad 1 \mathrm{~cm}$ positioning error of leaves on several scenarii using the $R$ option. Errors are generated using $a$ uniform distribution (dark grey) or Student's distribution (light grey). The STAR values from the original tree are taken as the reference.

Positioning errors in plant organs may also affect the convex envelope of plant components. To assess the impact of this phenomenon on the STAR computation using scenarii with option $\mathrm{R}$, we created a set of 5 mango mock-ups by introducing a $1 \mathrm{~cm}$ error on leaf position with the previously described distributions. For each of these trees, integrated $S T A R$ values were computed for scenarii AAA, AAR, ARR, RRR and --R to assess the cumulative impact of option R. Results are shown in Fig. 5.1 b. with the $S T A R$ value of the all-A scenario of the original tree taken as reference. An increase in the STAR estimation error with the use of $\mathrm{R}$ option at different scales is clearly visible (the effect is slightly reduced for the Student's distribution), and is due to the fact that porosity of a component results from the product of its subcomponents 

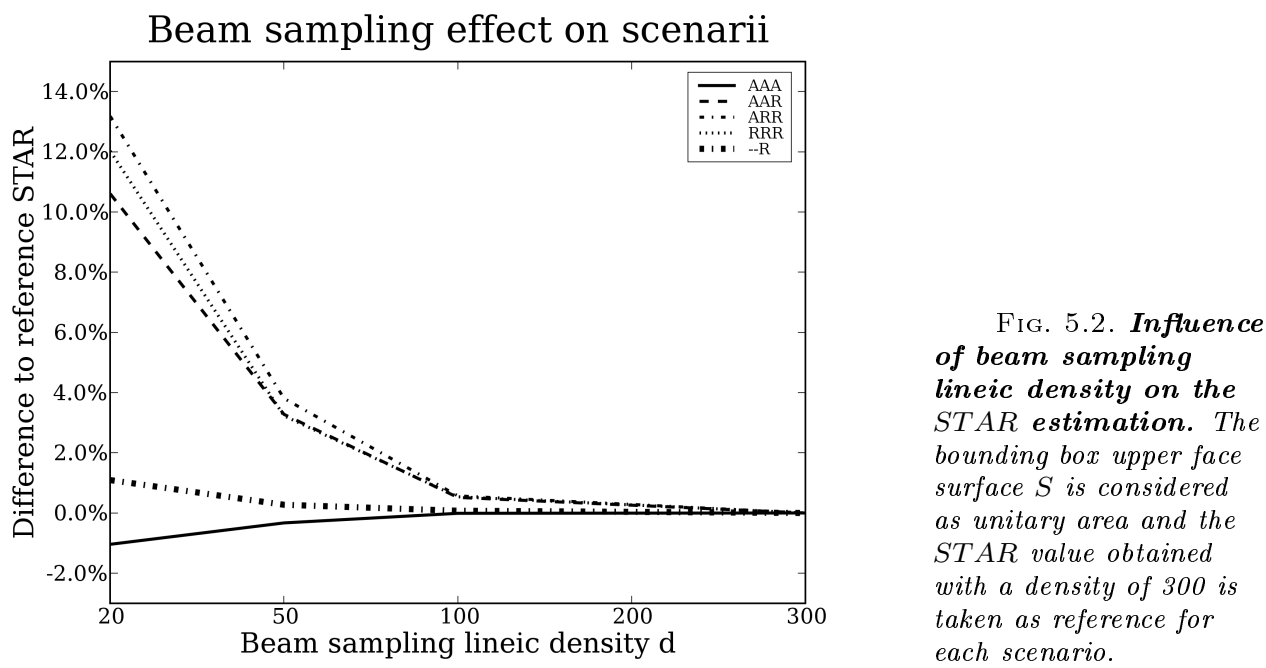

porosities, Eq.(2.8). Using other types of bounding envelopes like boxes, spheres or ellipsoids induces a larger envelope volume [2] and thus leads to similar error effects. If all-A scenario $S T A R$ value of the mock-up with leaf positioning errors is taken as the reference for the multiscale organisation analysis, then, we still find the same result trends.

Beam sampling effect. As mentioned in section 2.2, the density of the beam sampling may influence STAR estimation. The plant is included in a bounding box such that its upper face is orthogonal to the light direction, $\Omega$. This face is subdivided into cells of a regular grid whose centers define the locations of the beams. This defines a beam sampling density as $\frac{\mathcal{B}}{S}, \mathcal{B}$ being the total number of beams and $S$ the area of the upper face. In the case of our regular grid we control the sampling density by the lineic density of the beams, $d$, defined as

$$
d=\sqrt{\frac{\mathcal{B}}{S}},
$$

representing the number of beams per unit lenght along the grid axes. We assessed the effect of the sampling density by computing $S T A R$ values for several scenarii using different lineic densities: 20,50,100, 200 and 300, the latest being the one used to conduct the multi-scale analysis of section 4 . For each value of $d$, integrated $S T A R$ values were computed for scenarii AAA, AAR, ARR, RRR and --R. Results show that convergence was rapidly obtained with increasing density (the error was less than $0.5 \%$ for densities over 100), Fig. 5.2. Using a lineic density over 100 guarantees a good STAR estimation regardless of the used scenario. Moreover, since the complexity of our algorithm is quadratic in the beam sampling lineic density (see section 5.4 for details), large gain in computation time is achieved by decreasing $d$ from 300 to 100 with almost no loss of quality in the results.

5.3. A unifying approach. In section 2.2 , we observed that, surprisingly, the recursive equations (2.8) and (2.11) relating component porosities at different scales were actually identical despite the fact that they were derived from two different hypotheses. This raises the question as to whether these equations can be derived from a single unifying framework. To answer this question, let us call $\left\{x_{1} \ldots x_{n}\right\}$ 
the set of subcomponents of a component $c$, and $I_{b}(c)$ indicates whether the beam $b$ intersects with the envelope of $c\left(1=\right.$ yes, $0=$ no). Let us also denote $X_{b}(c)$ the random variable such that $X_{b}(c)=1$ if the beam $b$ is intercepted by an elementary component of $c$, i.e. it interacts with $c$, and $X_{b}(c)=0$ otherwise. With these definitions the porosity of a component $c$ for the beam $b$ is defined as

$$
p_{0}^{b}(c)=P\left(X_{b}(c)=0 \mid I_{b}(c)=1\right),
$$

reciprocally, its opacity is thus

$$
p^{b}(c)=1-p_{0}^{b}(c)=P\left(X_{b}(c)=1 \mid I_{b}(c)=1\right) .
$$

Let us now consider a beam $b$ that intersects a component c, i.e. $P\left(I_{b}(c)=0\right)=0$, thus the decomposition

$P\left(X_{b}(c)=0\right)=P\left(X_{b}(c)=0 \mid I_{b}(c)=1\right) P\left(I_{b}(c)=1\right)+P\left(X_{b}(c)=0 \mid I_{b}(c)=0\right) P\left(I_{b}(c)=0\right)$,

yields

$$
p_{0}^{b}(c)=P\left(X_{b}(c)=0 \mid I_{b}(c)=1\right)=P\left(X_{b}(c)=0\right) .
$$

A beam $b$ does not interact with a component $c$ if and only if $b$ does not interact with any subcomponent of $c$. Since going through a subcomponent without being intercepted does not modify the beam, all $P\left(X_{b}\left(x_{i}\right)=0\right)$ are independent and thus we have

$$
\begin{aligned}
P\left(X_{b}(c)=0\right) & =P\left(X_{b}\left(x_{1}\right)=0, \ldots, X_{b}\left(x_{n}\right)=0\right) \\
& =\prod_{k=1}^{n} P\left(X_{b}\left(x_{k}\right)=0\right)=\prod_{k=1}^{n}\left[1-P\left(X_{b}\left(x_{k}\right)=1\right)\right] .
\end{aligned}
$$

Using the fact that the probability for a beam $b$ to interact with a subcomponent that it is not intersecting, $P\left(X_{b}\left(x_{k}\right)=1 \mid I_{b}\left(x_{k}\right)=0\right)$, is obviously 0 , the decomposition of $P\left(X_{b}\left(x_{k}\right)=1\right)$ yields

$$
P\left(X_{b}\left(x_{k}\right)=1\right)=P\left(X_{b}\left(x_{k}\right)=1 \mid I_{b}\left(x_{k}\right)=1\right) P\left(I_{b}\left(x_{k}\right)=1\right) .
$$

Using expression Eq.(5.5) to replace $P\left(X_{b}\left(x_{k}\right)=1\right)$ in the component porosity equation, Eq.(5.4), and given the complementary relationship between opacity and porosity Eq.(5.3), the expression of opacity becomes

$$
P\left(X_{b}(c)=1 \mid I_{b}(c)=1\right)=1-\prod_{k=1}^{n}\left[1-P\left(X_{b}\left(x_{k}\right)=1 \mid I_{b}\left(x_{k}\right)=1\right) P\left(I_{b}\left(x_{k}\right)=1\right)\right],
$$

which illustrates our previous remark stating that opacity is controlled by two factors, first the opacity of the subcomponents : $P\left(X_{b}\left(x_{k}\right)=1 \mid I_{b}\left(x_{k}\right)=1\right)$, and second their spatial distribution : $P\left(I_{b}\left(x_{k}\right)=1\right)$. Indeed, when using option A, $P\left(I_{b}\left(x_{k}\right)=1\right)$ is either equal to 0 , (the beam does not intersect the component) or 1 , (the beam intersects the component). Therefore this term acts as a filter to disregard all subcomponents not intersected by the beam $b$. This is equivalent to Eq.(2.8) where the opacity of not intercepted subcomponents is equal to 0 . 
When using option $\mathrm{R}$ the spatial distribution of subcomponents can only be estimated. The probability of intersecting a subcomponent can be expressed as

$$
P\left(I_{b}\left(x_{k}\right)=1\right)=\frac{P E A^{b}\left(x_{k}\right)}{P E A^{b}(c)},
$$

whereas the expression of porosity of the subcomponent for the beam $b$ is

$$
P\left(X_{b}\left(x_{k}\right)=1 \mid I_{b}\left(x_{k}\right)=1\right)=\frac{P L A^{b}\left(x_{k}\right)}{P E A^{b}\left(x_{k}\right)} .
$$

Since $P E A^{b}(c)$ is the beam cross-section, $A_{b}$, the product of these two quantities in Eq.(5.6) yields the expression of the beam interception probability.

$$
\begin{aligned}
P\left(X_{b}\left(x_{k}\right)=1 \mid I_{b}\left(x_{k}\right)=1\right) P\left(I_{b}\left(x_{k}\right)=1\right) & =\frac{P L A^{b}\left(x_{k}\right)}{P E A^{b}\left(x_{k}\right)} \frac{P E A^{b}\left(x_{k}\right)}{P E A^{b}(c)} \\
& =\frac{P L A^{b}\left(x_{k}\right)}{A_{b}}
\end{aligned}
$$

which leads us to the second equation, Eq.(2.10), that was derived in case R. Consequently, we showed that it was possible to derive equations for both cases $A$ and $R$ from a unique expression, Eq.(5.6), that unify both situations.

5.4. Implementation issues and complexity. This software has been written in Python and $\mathrm{C}++$. It is a stand alone module part of the VPlants software project (successor of AMAPmod [16]).

In a first step a 3D shape is associated with each component of the MTG at each scale. The leaf geometry is defined using the PlantGL library [34] and convex hulls are computed with the QuickHull algorithm [4] available in this library. The multiscale computation of opacity is carried out for each direction $\Omega$ as follows. It starts with a double Z-buffer approach: two opposite orthographic cameras oriented along $\Omega$ direction are used to generate two images of each geometric component of the plant from the same distance. Each pair of facing pixels from the two orthographic views represents the same beam $b$ and its cross section area, $A_{b}$, is the area represented by a pixel expressed in metric units. The Z-values of each pixel yield the beam in- and out-points in the component. The travel distance of a beam within the envelope of component $j$ at scale $i, \mathcal{L}_{i, j}^{b}$, is deduced from these two values. This length is null for components associated with planar shape, e.g. leaves. Moreover, this double Z-buffer approach allows us to identify the beams intercepted by each component which is required for the use of option A, i.e. when actual component positions are used. In this approach convex envelopes are used since multiple in- and out-points are not taken into account, hence non-convex envelopes will be treated as convex ones. This step also provides the projected area of the component shape by multiplying $A_{b}$ with the number of pixels within the orthographic image. All these quantities are scenario-independent and thus are computed only once for each direction $\Omega$. The volume of a convex hull, $\mathcal{V}_{i, j}$, is computed separately using routines implemented in the PlantGL library [34].

Next the recursive scheme described in Fig.(2.3) is applied to compute the opacity according to the used scenario. The two recursive procedures used to compute the opacity of a component, $p_{i, j}$, and the beam opacity of a component, $p_{i, j}^{b}$, are described 
by Algorithms 1 and 2 respectively. The recursion starts from macroscopic components towards microscopic ones and ends when leaves are reached. It is important to note that in practice each value is computed once - the first time needed - and then stored for future use to save computation time.
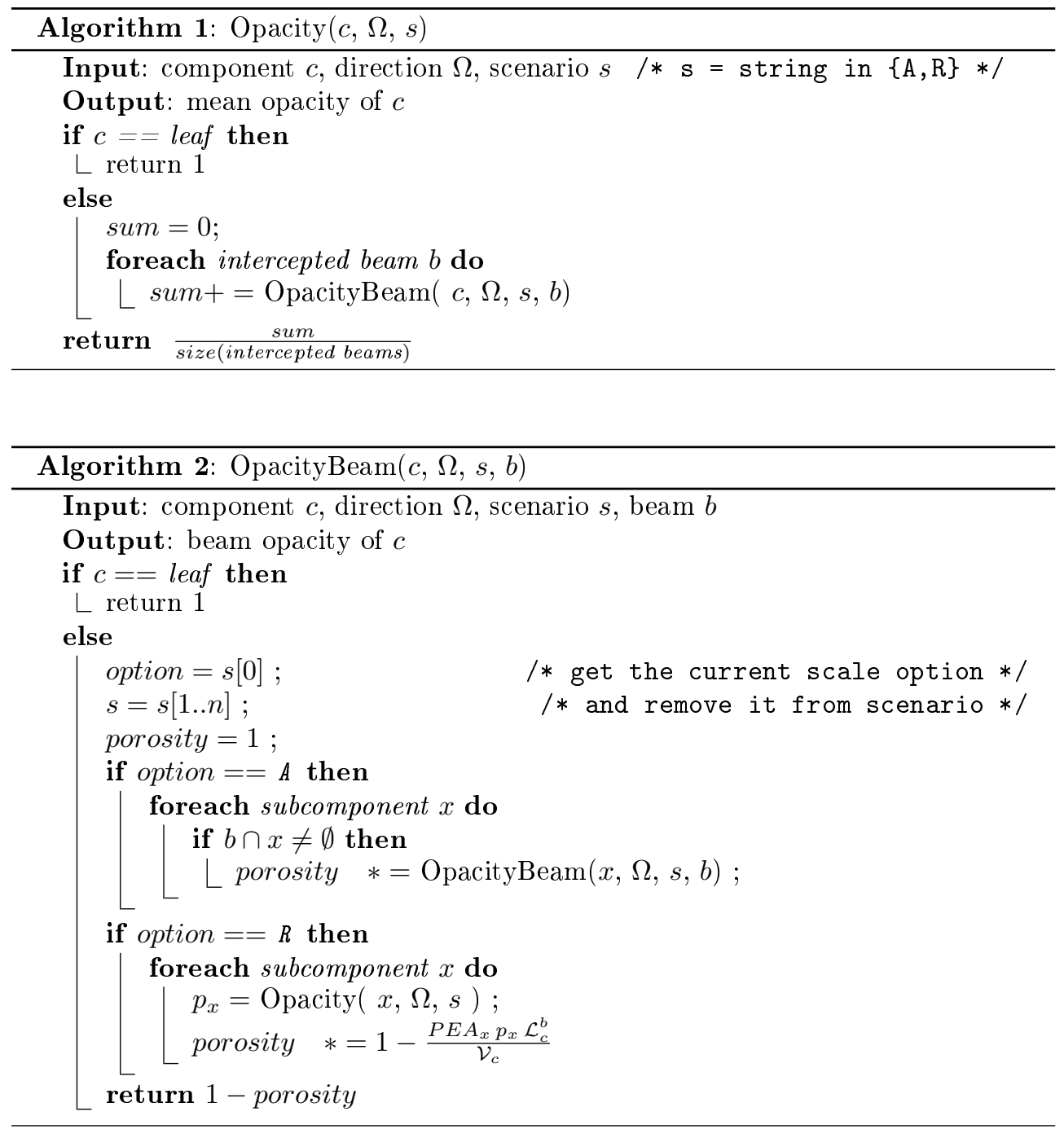

Leaves are considered as opaque components, thus their opacity does not need to be computed. The test whether a beam intersects a leaf or not is considered as the atomic operation. Therefore, the computation cost of one component opacity depends on the number of intersected beams, i.e. the number of pixels of its envelope projection in direction $\Omega$, and the number of its subcomponents. In the worst case scenario, every component is intersected by all beams. A very simple model allows us to evaluate the computation cost of our algorithm. Let us denote the number of scales by $k$ and let us assume that the number of subcomponents, $N$, is identical for all components at every scale. The total number of components is consequently

$$
1+N+N^{2}+\ldots+N^{k-1}=\mathcal{O}\left(N^{k-1}\right)=\mathcal{O}(n),
$$


where $n=N^{k-1}$ is the number of leaves, i.e. components of the last scale. Let us define a size ratio, $\delta$, by comparing the size of a component to the (smaller) size of its subcomponents. We also assume that the size ratio between two scale is constant and lesser than 1 , that is

$$
\forall i, j, l \quad \mathcal{V}_{i+1, j}=\delta^{3} \mathcal{V}_{i, l} \quad \text { and } \quad P E A_{i+1, j}=\delta^{2} P E A_{i, l}
$$

Let $B_{i}$ be the number of beams intercepted by a component at scale $i$ with $B_{0}=B$. As a consequence of a constant size ratio, the number of intercepted beams at one scale is related to the number of intercepted beams at the previous scale.

$$
B_{i+1}=\delta^{2} B_{i}=\delta^{2 i} B
$$

Consequently, the total cost $\gamma$ is the sum of each component cost at every scale except leaf scale

$$
\begin{aligned}
\gamma & =1 \times N B+N \times N B \delta^{2}+N^{2} \times N B \delta^{4}+\ldots+N^{k-2} \times N B \delta^{2(k-2)} \\
& =\mathcal{O}\left(N^{k-1} B \delta^{2(k-2)}\right)=\mathcal{O}\left(n B \delta^{2(k-2)}\right)
\end{aligned}
$$

The worst case is reached if $k=2$, thus the total cost is in $\mathcal{O}(n B)$. However, when $k>2$ since $\delta<1$ the gain in complexity due to the hierarchical structure is proportional to $\delta^{2(k-2)}$. For example, using the plant illustrated in Fig.2.1, adding 1 scale to the 2 basic scales will reduce the complexity by $\frac{1}{9}\left(\delta=\frac{1}{3}\right)$ and for 2 additional scales, the complexity will be reduced by $\frac{1}{81}$.

6. Conclusion. This paper presented a new framework to model efficiently light interception by isolated trees. The turbid medium approach, usually limited to large canopies because of its statistical description of plants, has been adapted to isolated trees. The modeling was based on a multiscale representation of plants and on a porous envelope hypothesis. We defined recursive expressions to compute the opacity of components with two types of spatial distribution hypotheses that can be chosen at each scale independently. The combination of these options defined scenarii that allowed us to analyze the influence of the plant architecture on light interception through the generalization of a dispersion parameter $\mu$ which expresses the departure of a plant foliage from randomness. This model was then assessed on 3D digitized peach and mango trees. Peach trees were markedly more clumped than mango trees. The two species showed different clumping behaviors but with the same trend for scaffold branches toward regular positioning. We showed that Oker-Blom and Kellomaki's partial grouping model can be used for mango trees but not for peach trees which shows a different type of clumping that does not fit their original assumption. Our model alleviate this problem by making it possible to use a variable number of scales and can be thus applied to both situations. Moreover the true $S T A R$ values were always comprise between the values from the full-grouping scenario and the partial grouping one disregarding the scaffold scale. This suggests that the multiscale organization is not the only factor involved in the light interception strategy of trees.

The proposed multiscale framework may be used to optimize plant architecture measurement in the context of modeling light interception by plants. It also defines a versatile and incremental procedure to compute light interception up to a desired level of accuracy, ranging from coarse descriptions, i.e. using the turbid medium hypothesis at plant scale, to detailed descriptions, e.g. obtained by $3 \mathrm{D}$ digitizing, with 
a series of intermediate options defined by the number of scales taken into account and their relative positioning. The multiscale organization of components represented by porous envelopes provides another advantage in terms of model flexibility, depending on the availability of field measurements the envelopes and their positions can either correspond to the actual field values or be infered from statistical assumptions.

Acknowledgements. We thank Yann Guédon for a thorough reading of the paper. We thank Gabriela Sonohat, Didier Combes and Vengadessan Kulandaivelu for their help to digitizing the peach trees. We are grateful to AIP and PFI programmes of INRA and to ATP Manguier programme from CIRAD for financial support of the digitizing process. 


\section{REFERENCES}

[1] B. Adam. Pol95 - software to drive a polhemus fastrak 3 space $3 \mathrm{~d}$ digitiser. Technical report, UMR PIAF INRA-UBP, Clermont-Ferrand, 1999. 11

[2] F. Boudon. Représentation géométrique de l'architecture des plantes. PhD thesis, Université Montpellier 2, 2004. 17

[3] F. Boudon, C. Godin, C. Pradal, O. Puech, and H. Sinoquet. Estimating the fractal dimension of plants using the two-surface method. an analysis based on 3d-digitized tree foliage. Fractals, 14(3), 2006. 4, 5

[4] C. Bradford-Barber, D. P. Dobkin, and H. Huhdanpaa. The quickhull algorithm for convex hulls. ACM Transactions on Mathematical Software, 22(4):469-483, December 1996. 19

[5] G. A. Carter and W. K. Smith. Influence of shoot structure on light interception and photosynthesis in conifers. Plant Physiology, 79(4):1038-1043, December 1985. 3

[6] E. Casella and H. Sinoquet. Botanical determinants of foliage clumping and light interception in two-year-old coppice poplar canopies: assessment from 3-d plant mock-ups. Annals of Forest Science, 64:395-404, 2007. 2

[7] A. Cescatti. Modelling the radiative transfer in discontinuous canopies of asymmetric crowns. ii. model testing and application in a norway spruce stand. Ecological Modelling, 101(2):275284, August 1997. 14

[8] D. A. Charles-Edwards and J. H. M. Thornley. Light interception by an isolated plant a simple model. Annals of Botany, 37(4):919-928, September 1973. 2, 8

[9] S. G. Chen, R. Ceulemans, and I. Impens. A fractal-based populus canopy structure model for the calculation of light interception. Forest Ecology and Management, 69(1-3):97-110, 1994. 13

[10] S. Cohen, M. Fuchs, S. Moreshet, and Y. Cohen. The distribution of leaf area, radiation, photosynthesis and transpiration in a shamouti orange hedgerow orchard. part ii. photosynthesis, transpiration, and the effect of row shape and direction. Agricultural and Forest Meteorology, 40(2):145-162, July 1987. 3

[11] S. Cohen, P. Mosoni, and M. Meron. Canopy clumpiness and radiation penetration in a young hedgerow apple orchard. Agricultural and Forest Meteorology, 76(3-4):185-200, October 1995. $2,13,14$

[12] P. Ferraro, C. Godin, and P. Prusinkiewicz. Toward a quantification of self-similarity in plants. Fractals, 2(12):91-109, 2005. 4

[13] J. D. Foley, A. van Dam, S. K. Feiner, and J. F. Hughes. Computer Graphics: Principles and Practice in C. Addison-Wesley Professional, second edition, August 1995. 1

[14] P. Giauque. Conduite du verger de pêcher. Recherche de la performance. CTIFL (Centre Technique Interprofessionnel eds Fruits et Legumes) Editions, 2003. 10

[15] C. Godin and Y. Caraglio. A multiscale model of plant topological structures. Journal of Theoretical Biology, 191(1):1-46, March 1998. 4

[16] C. Godin, E. Costes, and Y. Caraglio. Exploring plant topology structure with the amapmod software : an outline. Silva Fennica, 31(3):355-366, 1997. 19

[17] C. Godin, E. Costes, and H. Sinoquet. A method for describing plant architecture which integrates topology and geometry. Annals of Botany, 84(3):343-357, September 1999. 4, 12

[18] J. E. Jackson and J. W. Palmer. A simple model of light transmission and interception by discontinuous canopies. Annals of Botany, 44:381-383, September 1979. 3

[19] H. G. Jones. Plants and Microclimate: A Quantitative Approach to Plant Physiology. Cambridge University Press, 1992. 1

[20] D. S. Kimes and J. A. Kirchner. Radiative transfer model for heterogeneous 3-d scenes. Applied Optics, 21(22):4119+, 1982. 3

[21] Y. Knyazikhin, G. Mießen, O. Panfyorov, and G. Gravenhorst. Small-scale study of threedimensional distribution of photosynthetically active radiation in a forest. Agricultural and Forest Meteorology, 88(1-4):215-239, December 1997. 3

[22] J. E. Mann, G. L. Curry, and P. J. H. Sharpe. Light interception by isolated plants. Agricultural Meteorology, 20(3):205-214, June 1979. 2, 8

[23] M. Monsi and T. Saeki. Uber den lichtfaktor in den pflanzengesellschaften und seine bedeutung fur die stoffproduktion. Japanese Journal of Botany, 14(1):22-52, 1953. 2

[24] B. Moulia and H. Sinoquet. Crop structure and light microclimate: Characterization and applications, chapter Three-dimensional digitizing systems for plant canopy geometrical structure : a review, pages 183-193. INRA, 1993. 15

[25] R. B. Myneni. Modeling radiative transfer and photosynthesis in three-dimensional vegetation canopies. Agricultural and Forest Meteorology, 55(3-4):323-344, June 1991. 3 
[26] U. Niinemets, A. Cescatti, and C. Rochelle. Constraints on light interception efficiency due to shoot architecture in broad-leaved nothofagus species. Tree Physiology, 24:617-630, 2004. 2

[27] T. Nilson. A theoretical analysis of the frequency of gaps in plant stands. Agricultural Meteorology, 8:25-38, 1971. 2, 10

[28] J. M. Norman and P. G. Jarvis. Photosynthesis in sitka spruce (picea sitchensis (bong.) carr.): V. radiation penetration theory and a test case. The Journal of Applied Ecology, 12(3):839878, 1975. 3

[29] J. M. Norman and J. M. Welles. Radiative transfer in an array of canopies. Agronomy Journal, $75: 481-488,1983.14$

[30] P. Oker-Blom and S. Kellomaki. Effect of grouping of foliage on within-stand and withincrown light regimes: comparison of radiation and grouping canopy models. Agricultural Meteorology, 28:143-155, 1983. 3, 9, 14, 15

[31] P. Oker-Blom and H. Smolander. The ratio of shoot silhouette area to total needle area in scots pine. Forest Science, 34(4):894-906, December 1988. 3

[32] J. Phattaralerphong, J. Sathornkich, and H. Sinoquet. A photographic gap fraction method for estimating leaf area of isolated trees: Assessment with 3d digitized plants. Tree Physiology, 26(9):1123-1136, September 2006. 8

[33] Polhemus Inc. Colchester, VT, USA. 3SPACE FASTRAK User's Manual, Revision F., 1993. 11

[34] C. Pradal, F. Boudon, C. Nouguier, J. Chopard, and C. Godin. Plantgl : a python-based geometric library for $3 \mathrm{~d}$ plant modelling at different scales. Technical report, INRIA, November 2007. 19

[35] P. Prusinkiewicz. Self-similarity in plants: Integrating mathematical and biological perspectives. In Thinking inPatterns. Fractals and Related Phenomena in Nature., pages 103-118. Novak, M., 2004. 4

[36] J. Ross. The Radiation Regime and Architecture of Plant Stands. The Hague-Boston-London : Dr. W. Junk, 1981. 1, 2, 5

[37] H. Sinoquet, J. Phattaralerphong, P. Kasemsap, and S. Thanisawanyangkura. Light and carbon acquisition partitioning between flushes of two-year-old mango trees. In 4 th Workshop on Functional-Structural Plant Models (FSPM04), pages 146-149. CIRAD, 2004. 9

[38] H. Sinoquet and P. Rivet. Measurement and visualization of the architecture of an adult tree based on a three-dimensional digitising device. Trees - Structure and Function, 11(5):265270, April 1997. 1, 2, 4, 12

[39] H. Sinoquet, G. Sonohat, J. Phattaralerphong, and C. Godin. Foliage randomness and light interception in 3-d digitized trees: an analysis from multiscale discretization of the canopy. Plant, Cell \& Environment, 28(9):1158-1170, September 2005. 3, 7

[40] H. Sinoquet, J. Stephan, G. Sonohat, P. E. Lauri, and P. Monney. Simple equations to estimate light interception by isolated trees from canopy structure features: assessment with threedimensional digitized apple trees. New Phytologist, 175(1):94-106, July 2007. 3, 10

[41] H. Sinoquet, S. Thanisawanyangkura, H. Mabrouk, and P. Kasemsap. Characterization of the light environment in canopies using $3 \mathrm{~d}$ digitising and image processing. Annals of Botany, 82(2):203-212, August 1998. 1, 11

[42] G. Sonohat, H. Sinoquet, V. Kulandaivelu, D. Combes, and F. Lescourret. Three-dimensional reconstruction of partially 3d-digitized peach tree canopies. Tree Physiology, 26:337-351, 2006. $10,12,15$

[43] S. Thanisawanyangkura, H. Sinoquet, P. Rivet, M. Cretenet, and E. Jallas. Leaf orientation and sunlit leaf area distribution in cotton. Agricultural and Forest Meteorology, 86(1-2):1-15, August 1997. 15

[44] L. Urban, X. Le Roux, H. Sinoquet, S. Jaffuel, and M. Jannoyer. A biochemical model of photosynthesis for mango leaves: evidence for the effect of fruit on photosynthetic capacity of nearby leaves. Tree Physiology, 23:289-300, 2003. 10

[45] F. J. Villalobos, F. Orgaz, and L. Mateos. Non-destructive measurement of leaf area in olive (olea europaea 1.) trees using a gap inversion method. Agricultural and Forest Meteorology, 73(1-2):29-42, February 1995. 8

[46] D. Whitehead, J. C. Grace, and M. J. Godfrey. Architectural distribution of foliage in individual pinus radiata $d$. don crowns and the effects of clumping on radiation interception. Tree Physiology, 7(1 23 4):135-155, December 1990. 2, 13

[47] M. Willaume, P. E. Lauri, and H. Sinoquet. Light interception in apple trees influenced by canopy architecture manipulation. Trees, 18(6):705-713, November 2004. 13 